\title{
Safe Opioid Prescription: A SMART on FHIR Approach to Clinical Decision Support
}

\author{
Shyamashree Sinha1,2, Mark Jensen ${ }^{1,3}$, Sarah Mullin", Peter L Elkin ${ }^{1}$ \\ 1. Department of Biomedical Informatics, University at Buffalo State University of New York \\ 2. Department of Anesthesiology, University at Buffalo State University of New York \\ 3. Department of Philosophy, University at Buffalo State University of New York
}

\begin{abstract}
Background

Prescription opioid pain medication overuse, misuse and abuse have been a significant contributing factor in the opioid epidemic. The rising death rates from opioid overdose have caused healthcare practitioners and researchers to work on optimizing pain therapy and limiting the prescriptions for pain medications. The state of New York has implemented a prescription drug monitoring program(PDMP), amended public health law to limit the prescription of opioids for acute pain and utilized the resources of the state and county health departments to help in curbing this epidemic. The recent publication of guidelines for prescription opioids from CDC [1] and ASIPP (American Society of Interventional pain practitioners) have independently reviewed literature and found good evidence of limiting opioid prescription for acute and chronic non cancer pain. [2]

Method

Over the last decade, advanced technology has increased the complexity of electronic health records systems leading to the development of Clinical Decision Support Systems (CDSS) to aid the work flow of healthcare providers. There are several systematic reviews on the effectiveness and utility of CDSSs. A common consensus is that commercially and locally developed CDSS are effective in improving patient measures while actual workload improvement and efficient cost-cutting measure are not significantly improved by CDSS. Patient provider involvement in developing CDSS is a determinant of its success and utilization rates. [7] Therefore, a plug and play form of CDSS which can be implemented from an external platform through secure channels would be more effective.
\end{abstract}

Design

The Health Level Seven's (HL7) open licensed interoperability standard Fast Health Interoperability Resources (FHIR) has a platform, Substitutable Medical Applications and Reusable Technologies (SMART) for CDSS app development by a third party. [3] We adopted these open source standard to plan to develop an app for accessible and efficient implementation of the recently published guidelines for management of pain with prescription opioid medications. 


\section{Aim}

The goals for this CDSS tool are to achieve proper monitoring of prescription drugs, patients' medication list and potential interactive medications, surveillance for abuse/ misuse, patient involvement in alternative therapy, reporting problems and obtaining adequate pain control. The final step would be to implement the system in clinical practice and to monitor usage rates and measure the outcomes of successful or unsuccessful implementations.

DOI: $10.5210 /$ ojphi.v9i2.8034

\section{Copyright @2017 the author(s)}

This is an Open Access article. Authors own copyright of their articles appearing in the Online Journal of Public Health Informatics. Readers may copy articles without permission of the copyright owner(s), as long as the author and OJPHI are acknowledged in the copy and the copy is used for educational, not-for-profit purposes.

\section{Introduction}

The mortality rate due to Opioid overdose has been consistently rising over the last decade, reaching epidemic proportions. New York State, including Western New York, has had a steady rise in opioid related deaths from 2014 to 2015: natural and semisynthetic opioid overdose death rose by $13.3 \%$, methadone related deaths rose by $9.1 \%$, and synthetic opioid other than methadone related deaths rose by $135.7 \%$ [1]. There is evidence that overuse, misuse. and abuse of prescription opioid pain medication is one of the major contributing factors in the rising death toll. This reasoning led to the publication of the recent CDC guidelines for prescription [2].

The population health problem due to prescription opioid use, abuse and dependence has been progressively worsening over the last two decades. Clinicians are faced with the challenge of treating pain adequately to improve quality of life while trying to prevent the potential of overuse, abuse and dependence among patients who are being treated by prescription opioid medications. Many studies have shown narcotic pain medications are known to decrease pain threshold and increase the need for pain medications [3].

The need for clinical decision support for pain control and monitoring of patients' medication has led to many attempts at developing tools based on established guidelines. The ASSIP recently published a two-part guideline on pain control which gives a stepwise approach to pain medication management and adequate pain control. This guideline states opioid pain medication therapy should only be limited to patients with intractable chronic pain who have shown demonstrable improvement with therapy [4].

Recently, the Centers for Disease Control and Prevention published a guideline for chronic opioid prescription based on a systematic review of current literature in 2016 [2]. These practice guidelines provide clinicians with a useful tool for making decisions of optimizing the use of prescription pain medications. However, the attempts at implementation of these guidelines in the form of applications have had limited outcomes that have been discussed later. 
Clinical Decision Support Systems (CDSS) based on Electronic Health Records have been evolving for more than a decade. A paper published by the federal health IT unit outlines the need, importance and available technology behind the evolving CDSS tools [5].

Based on their recommendations a stepwise process of designing a CDSS would be to:

1. Identify and quantify a 'high-priority gap' in clinical quality between current outcomes and a stated goal. 'High priority' is the need for quality of care improvement aligns with the strategic goals and treatment outcomes that the health care organization is aiming for.

2. Map the workflow by which the clinical care pertaining to the target issue is delivered.

3. Design a future state workflow that includes the best ideas for closing the gap and eliminates as much waste as possible.

4. Identify the information necessary to support the future state workflow. Then, design, test, and perfect CDS interventions using the Four Rights- Right Info, Right Person, Right Medium and Right Format.

Our goal is the creation of an application (app) based decision support tool that is high priority in terms of population health needs, minimizes the consumption of time for the provider, and is more interactive for the patient. In addition, this app could be utilized as an aide in implementation of the goals of precision medicine, focusing on patient-centered care.

Therefore, in line with these goals, we propose the creation of the application OPIacutepain that follows the opioid prescribing guidelines set forth by the CDC to aide in the efficient and accurate prescription of opioid medication for acute pain.

\section{Background}

\subsection{Justification for implementation of a CDSS for prescription of Opioid pain} medication

The state of New York has recently implemented an amendment of the NY public health law (NYPHL 3331) limiting the number of days of prescription for opioid pain medication to not more than seven days for any acute non-cancer/non-terminal illness pain [6]. Due to the rising death rates from opioid overdose, the CDC's prescription opioid pain medication guidelines are aimed at curbing opioid dependence, abuse and misuse of pain medications. The patients on opioid pain medications for chronic pain are required to be monitored by Prescription Drug Monitoring Programs (PDMPs) and regular urine screening. Applying the proper CDSS implementation to prescription opioid pain medications, the five steps are as follows [2]:

- High Priority gap in the clinicians' prescription of pain medication for acute pain conditions. The amendment of state law and CDC guidelines all aim at cutting down on that acute pain prescriptions.

- The work flow of prescription of pain medication starts as follows-

$>$ with the patient being aware of the other options for pain control 
$>$ then, deciding upon a treatment goal and working towards fulfilment

$>$ followed by an evidence or guideline based approach to prescribing pain medications

- Design a CDSS based on the latest technology. In order to be effective, the CDSS for opioids should be interactive and less invasive. Which means it can be used by the providers on demand and would be able to pull the right information for the patient in order to aid the clinician with decision making

- The SMART on FIHR app would be written based on the current guidelines from CDC and ASIPP.

\subsection{Review of Current Opioid-related Apps and CDS systems}

The number of attempts at developing CDSS has resulted in an aversion to yet another system. The clinicians and healthcare providers look at it as more of a deterrent in their work flow than any help [7] [5]. However, different technological approaches may be able to help in finding an answer to the complexity of electronic health records. The need for development of newer CDSS were initially based on national stimuli for EHR adoption, Meaningful Use requirements, Medicare Access and CHIP Re Authorization Act(MACRA) and Merit-based Incentive Payment System(MIPS). The rise of precision medicine, genomics and dynamic changes in the approach to medical care increased the complexity of care needed more emphasis on coordination of care. The increasing cost of medical care, patient empowerment/awareness and technological push for App culture are also drivers for development of newer CDSS [7] [5].

There are many opioid conversion calculators and morphine equivalency calculators on the market. However, each one has issues as a point of care tool, whether a hard to use interface, requiring the internet to download guidelines, having no citations for evidence based assessment, or requiring many plug in values that could be automatically taken from the electronic health record (EHR) or electronic medical record (EMR). Especially when it comes to conversion, many of the apps are not reliable. A recent study assessed the output variability and professional medical involvement in the authorship of 23 different apps [8]. 52\% of the apps had no stated medical professional involvement and $48 \%$ of the apps provided references for opioid conversion ratios [8].

The CDC recently released on app summarizing the 2016 guidelines. CDC Opioid Prescribing Guideline 2016 contains a morphine equivalency calculator, brief synopses of recommendations from the guidelines, glossary and a section on how to perform motivational interviewing with pain patients. The app includes links to full sections of the CDC guideline and references, but requires an internet connection. The MME calculator, which allows for multiple drugs to be calculated for a total MME/day, is built in and does not require internet connection and provides an alert when $\mathrm{MME} / \mathrm{day} \geq 90$. The alert suggests referring to a specialist and scheduling reassessment at least every 3 months, as well as suggesting recommendations through proper links in the app [9].

The $\mathrm{pH}$ Medical Opioid Converter App by Philip Eagan from the iTunes app store, use 2016 CDC guideline for Prescribing Opioids for Chronic Non-Cancerous Pain patients. The app includes an easy to use morphine equivalence calculator and an opioid to opioid calculator, using generic or trade names. Although the app has a simple to use interface and is based on current recommendations, it is the only one that comes at a cost (\$1.99), it does not have an Android 
counterpart, and very little information can be found about the author. In addition, like the CDC app, the guidelines and links are not built into the app and therefore, an internet connection is required [10].

The Safe Opioids app was developed with support from the Substance Abuse and Mental Health Services Administration through the Prescribers' Clinical Support System for Opioid Therapies. It includes four categories: Evaluation, Manage, Discontinue, and other online Resources. Under Evaluation, there is information on the assessment of pain, linking to an outside the app pdf, the Opioid Risk Tool, and links to drug prescription monitoring programs. Under manage, there is information on opioid management including links to medical guidelines on clinical use in treatment of chronic pain (e.g.: VA/DoD Clinical Practice Guidelines) from 2010. There is also a list of common side effects and advise for talking to patients about opioid abuse. There are also links to a tool for assessing depression. The app provides citations, showing how they are using evidence based medicine for decisions, however, the app is outdated and the citations have not been updated since 2010 [11].

OpioidCalc NYC, developed by The New York City Department of Health and Mental Hygiene (DOHMH), calculates MME based on the guidelines developed with the help of the CDC that outline key principles of safe and judicious prescribing practices, including high dosage defined as $M M E /$ day $\geq 100$. The app provides citations for evidence-based practice decisions. The app allows users to quickly and easily calculate the total daily MME a patient is taking, based on type of opioid analgesic, strength, and quantity. Multiple types of opioid analgesics can be included in the total daily MME calculation. An alert is displayed for total daily MME greater than or equal to 100 , indicating an increased risk for overdose. This alert also suggests reassessing the patient's pain status and treatment plan and provides a link to the DOHMH opioid-prescribing guidelines for additional information. It does not include I.V. formulations of medications and does not align with current CDC guidelines [12].

Opioid pain medication documentation and surveillance system (PODS) from electronic health records is one of the early attempts at medication management and patient education efforts. Like many other apps included in the electronic health records it was time intensive and cumbersome due to the fact that it was not interactive with the rest of the ATHENA EHR-Opioid Therapy for chronic non-cancer pain pop-up [13]. There were many good features in this system like patient safety features and some early warnings. The pop up in the EHR had a patient identifier on graphic user interphase, caution window where the patients risk factors against prescribing pain medication were mentioned, the treatment options, data tables, treatment checklist and information for researchers would appear in separate sections of the window. The dosage calculator and other options were present in a dropdown section.

The usability testing of this CDSS showed the lack of provider education, confusion in dosing calculations and titration schedules, access to relevant patient information, provider discontinuity, documentation, and access to validated assessment tools. Clinicians reported the time constraints in completing each prescribing decision and effective pain management based on guidelines. The figure below shows the pop up window. 


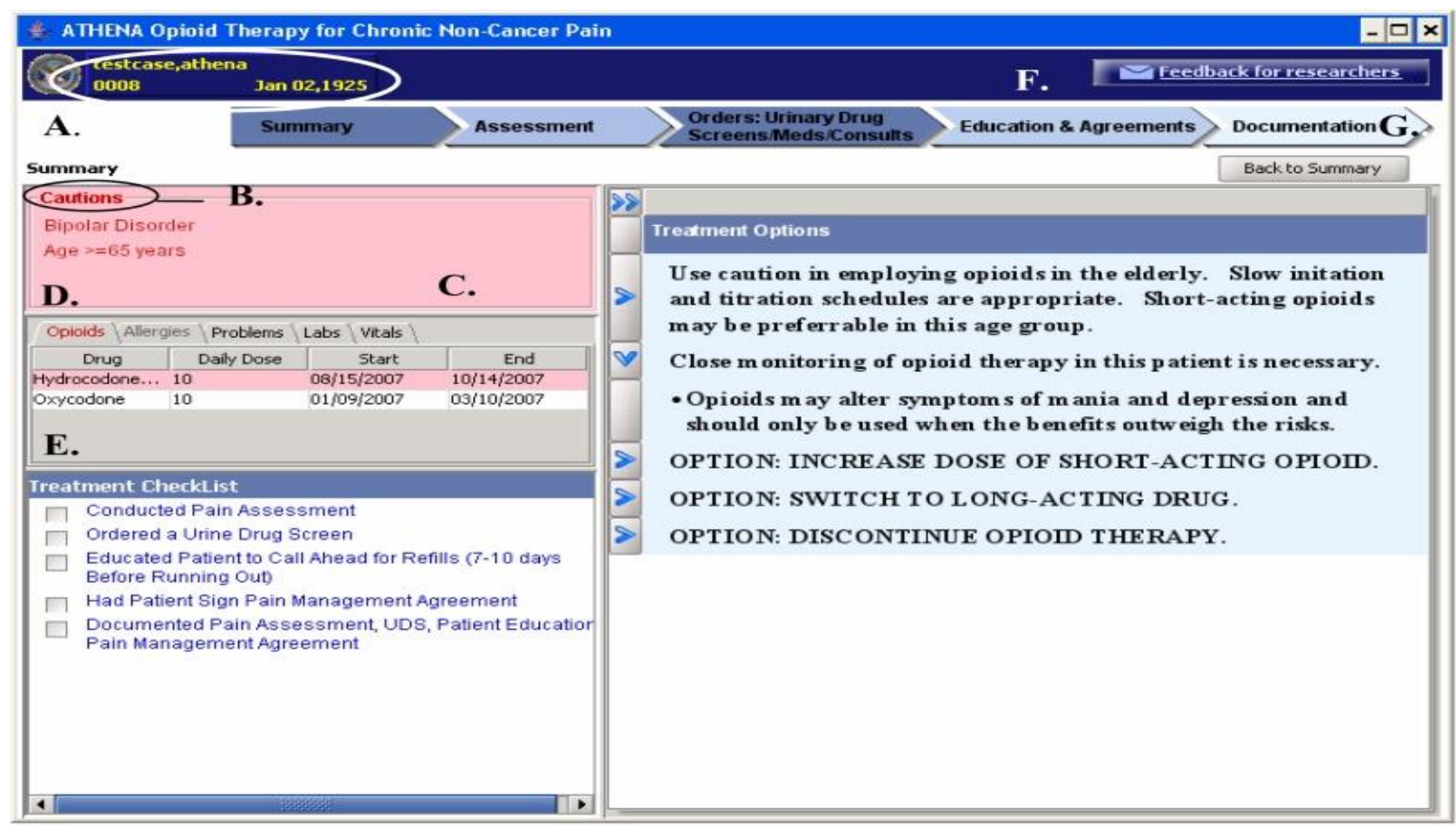

Figure 1. GUI Source: https://www.ncbi.nlm.nih.gov/books/NBK43756/figure/advancesmichel_92.f1/?report=objectonly

[Int J Med Inform. 2015 Apr;84(4):248-62. doi: 10.1016/j.ijmedinf.2015.01.004.

Epub 2015 Jan 17.]

\section{Proposed Solution}

The SMART on FHIR platform for interactive and integrated CDSS development is an open source resource which will be used to implement CDC guidelines with an aim to cut down the rate of opioid prescription for acute pain. The third party app development process aids the wide scale utilization of the app in FHIR compatible EHR environment. This is a process of using cell phone technology in building a more dynamic (can be used by healthcare providers and patients through different portals) CDSS tool that is user friendly and does not have to be built in as a part of the EHR system.

The SMART app will be independently placed in an APP library from where it can be accessed by the clinician while making the decision for treatment of pain.

Figure 2. explains the flow of the platform. 


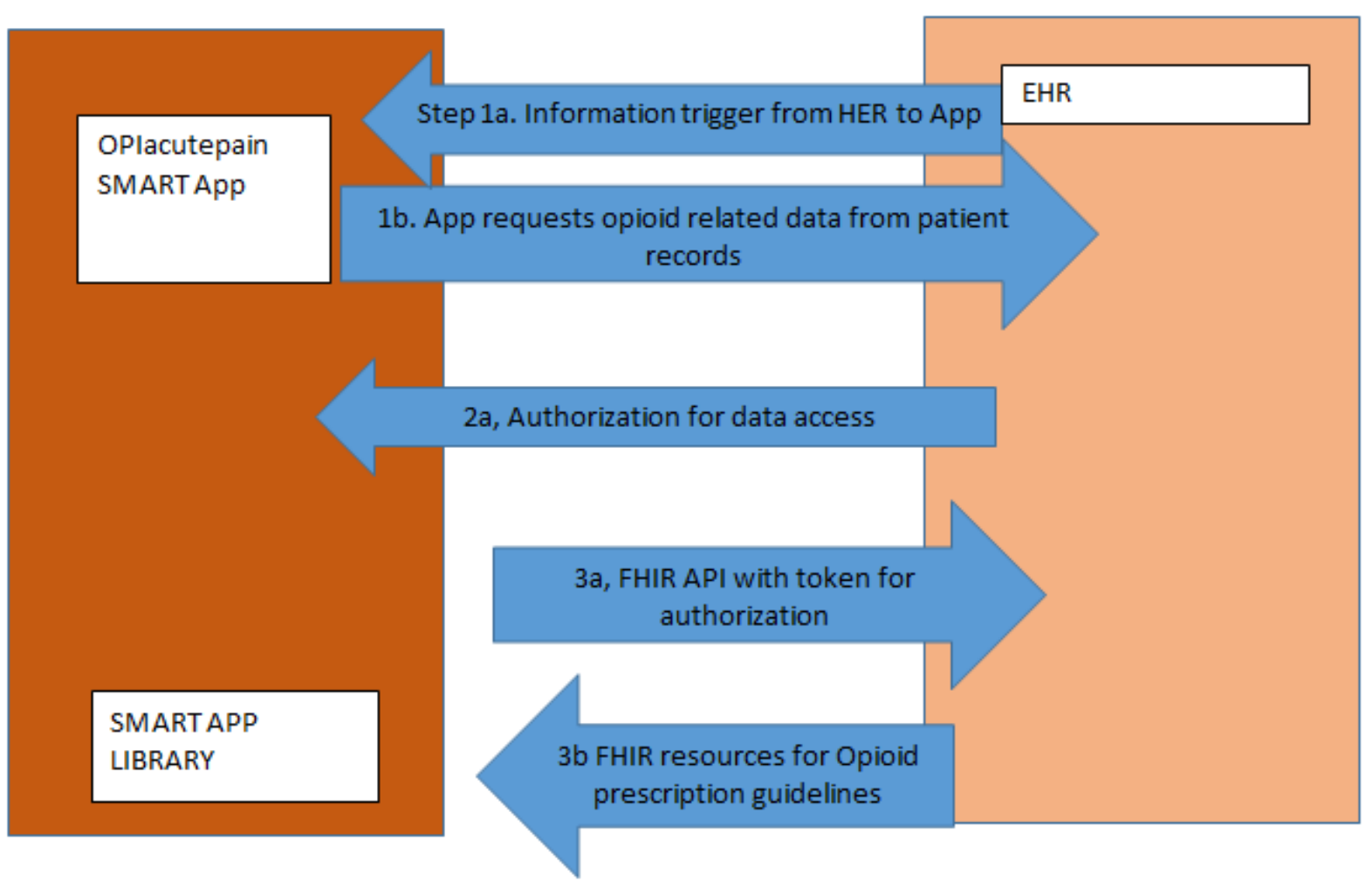

Figure 2. Flow diagram for OPIacutepain a SMART on FHIR approach for prescription opioids for acute pain

The CDC guidelines published in 2016 [2] were aimed at providing a decision making tool for clinicians, nurses and other healthcare providers to aid in the treatment of chronic and acute pain. The following chart (Figure 3) shows the different categories of pain the patients may present with and how the clinician may make their decision.

\begin{tabular}{|l|l|l|l|}
\hline Acute pain (first) & $\begin{array}{l}\text { Acute pain (prior } \\
\text { history) }\end{array}$ & Acute on chronic pain & Chronic pain on opioid \\
\hline $\begin{array}{l}\text { Inform physician of } \\
\text { treatment goals for } \\
\text { pain(Recommendation } \\
\text { 2) }\end{array}$ & $\begin{array}{l}\text { Inform physician of } \\
\text { treatment goals for } \\
\text { pain(Recommendation } \\
\text { 2) }\end{array}$ & $\begin{array}{l}\text { Inform physician of } \\
\text { treatment goals for } \\
\text { pain(Recommendation } \\
\text { 2) }\end{array}$ & $\begin{array}{l}\text { Inform physician of } \\
\text { treatment goals for } \\
\text { pain(Recommendation } \\
\text { 2) }\end{array}$ \\
\hline $\begin{array}{l}\text { Non pharmacologic } \\
\text { (rec 1) }\end{array}$ & $\begin{array}{l}\text { Assess Baseline pain } \\
\text { and function- PEG } \\
\text { assessment scale (see } \\
\text { Appendix A) }\end{array}$ & $\begin{array}{l}\text { Assess Baseline pain } \\
\text { and function- PEG } \\
\text { assessment scale (see } \\
\text { Appendix A) }\end{array}$ & $\begin{array}{l}\text { Assess Baseline pain } \\
\text { and function- PEG } \\
\text { assessment scale (see } \\
\text { Appendix A) }\end{array}$ \\
\hline
\end{tabular}




\begin{tabular}{|l|l|l|l|}
\hline $\begin{array}{l}\text { Alerts: history of } \\
\text { mental illness, Alcohol } \\
\text { or other substance } \\
\text { abuse, smoking }\end{array}$ & $\begin{array}{l}\text { Alerts: history of } \\
\text { mental illness, Alcohol } \\
\text { or other substance } \\
\text { abuse, smoking }\end{array}$ & $\begin{array}{l}\text { Alerts: history of } \\
\text { mental illness, Alcohol } \\
\text { or other substance } \\
\text { abuse, smoking }\end{array}$ & $\begin{array}{l}\text { Alerts: history of } \\
\text { mental illness, Alcohol } \\
\text { or other substance } \\
\text { abuse, smoking }\end{array}$ \\
\hline
\end{tabular}

Figure 3. Categories of Pain and Recommendations from CDC guidelines

The characteristics of the SMART Application that we propose are as follows:

The Application is aimed at providing interactive EHR based guidelines for acute pain patients where non pharmacologic, non-opioid pain medications are tried first followed by a short term opioid pain medication therapy.

The potential for fatal overdose may be there if the patient is on Benzodiazepines while being on opioid pain medication. Concurrent benzodiazepine usage may lead to fatalities in $31 \%-61 \%$ of the total overdose deaths [1]. This could be prevented by the interactive application.

In addition, the initial daily dose of opioids should not exceed $50 \mathrm{MME} /$ day and could go up to a maximum of $90 \mathrm{MME}$ per day. For dosages from 50 to $100 \mathrm{MME} /$ day, risk for overdose increased by a factor of 1.9 to 4.6 compared to dosages of 1 to $20 \mathrm{MME} /$ day. Greater than $100 \mathrm{MME} / \mathrm{day}$ increased by a factor of 2 to 8.9 compared to 1 to $20 \mathrm{MME} /$ day [1]. Risk for serious harm on higher dosage of opioid outweighs the treatment benefits.

The app will include a medication list of all the opioids a patient is currently using, and if there are opioids present, the conversion chart to calculate total MME/day produced by the CDC will be used to automatically calculate the total for the clinician (Figure 4).

Calculating morphine milligram equivalents (MME)
\begin{tabular}{|l|c|}
\hline OPIOID (doses in mg/day except where noted) & CONVERSION FACTOR \\
\hline Codeine & 0.15 \\
\hline Fentanyl transdermal (in mcg/hr) & 2.4 \\
\hline Hydrocodone & 1 \\
\hline Hydromorphone & 4 \\
\hline Methadone & \\
\hline $1-20$ mg/day & 4 \\
\hline $21-40$ mg/day & 8 \\
\hline $41-60$ mg/day & 10 \\
\hline$\geq 61-80$ mg/day & 12 \\
\hline Morphine & 1 \\
\hline Oxycodone & 1.5 \\
\hline Oxymorphone & 3 \\
\hline
\end{tabular}

Figure 4. CDC Calculating MME Conversion Factor chart 
Finally, the app will check if a urine drug screen (UDS) has been ordered. The CDC guidelines state, that a UDS provides the ability to "identify patients who might be at higher risk for opioid overdose or opioid use disorder, and help determine which patients will benefit from greater caution and increased monitoring or interventions when risk factors are present [1]. Depending on the outcome of medications and observations, recommendations will be provided that include counseling, doing the PEG-assessment, ordering a UDS, not prescribing opioid medications, tapering of opioid medications, and checking the state prescription drug monitoring program (PDMP) data. An outline of the workflow of the proposed app is included in Figure 5.

\section{Methods}

\subsection{FHIR}

FHIR $®$ (Fast Healthcare Interoperability Resources) is a next generation standards framework that builds on Health Level Seven International (HL7), an ANSI-accredited standard developing organization which provides standards for the exchange, integration, sharing, and retrieval of electronic health information supporting clinical practice and management. According to the FHIR standards, it "leverages existing logical and theoretical models to provide a consistent, easy to implement, and rigorous mechanism for exchanging data between healthcare applications". [14] In order to assure alignment with the current HL7 standards and interoperability, FHIR has builtin mechanisms for traceability to the HL7 RIM [14].

The basic building blocks of FHIR are called resources which share a set of characteristics, including a definition, a common set of metadata, and a human readable part [14-16]. Table 1 provides the list of resources and their elements that are of interest to the application. Following Meaningful Use Stage 2 criteria, resources rely on ontologies and terminologies, specifically SNOMED-CT, LOINC, RxNorm [14]. Therefore, our CDSS is ontology and terminology driven.

Currently, FHIR version 1.0.2 is supported by Epic, Cerner, and Allscripts Professional, three prominent electronic health record (EHR) systems [7]. For instance, according to the Open Epic website, Epic's integration works with FHIR 1.0.2 (DSTU2). With this specification, Epic supports retrieving data for most of the top-level resources such as "patient", "observation", "procedure", "medication." Since these are primarily the resources needed for our proposed app, this app could seemingly be used any system equipped with Epic, Cerner, or Allscripts professional and can easily be accessed through their web services and other methods, such as relational databases. 
Table 1. FHIR resources used for OPIacutepain

\begin{tabular}{|c|c|c|}
\hline Resource Type: & & Patient \\
\hline \multirow{2}{*}{$\begin{array}{l}\text { Definition: } \\
\text { Elements }\end{array}$} & \multicolumn{2}{|c|}{$\begin{array}{l}\text { The Patient Resource provides general demographic information about a } \\
\text { person receiving health care services from a specific organization. }\end{array}$} \\
\hline & Definition & Use in App \\
\hline Patient.name.given & First name & $\begin{array}{l}\text { Used to Make Sure you are querying correct } \\
\text { patient }\end{array}$ \\
\hline Patient.name.family & Last name & $\begin{array}{l}\text { Used to Make Sure you are querying correct } \\
\text { patient }\end{array}$ \\
\hline Patient.birthdate & birthdate & $\begin{array}{l}\text { Used to Make Sure you are querying correct } \\
\text { patient }\end{array}$ \\
\hline Patient.gender & gender & $\begin{array}{l}\text { Used to Make Sure you are querying correct } \\
\text { patient }\end{array}$ \\
\hline Resource Type: & \multicolumn{2}{|r|}{ MedicationOrder } \\
\hline Definition: & \multicolumn{2}{|c|}{$\begin{array}{l}\text { An order for both supply of the medication and the instructions for } \\
\text { administration of the medication to a patient. }\end{array}$} \\
\hline Elements & Definition & Use in App \\
\hline MedicationOrder.Status & $\begin{array}{l}\text { Lifecycle of prescription: want } \\
\text { active not draft }\end{array}$ & \\
\hline MedicationOrder.dateEnded & $\begin{array}{l}\text { Date when prescription was } \\
\text { stopped }\end{array}$ & Used to filter in only active/current prescriptions \\
\hline MedicationOrder.patient & $\begin{array}{l}\text { A link to a resource representing } \\
\text { the person to whom the } \\
\text { medication will be given. }\end{array}$ & Medication for specific patient \\
\hline
\end{tabular}




\begin{tabular}{|c|c|c|}
\hline MedicationOrder.medicationCodeableConcept & $\begin{array}{l}\text { Identifies the medication being } \\
\text { administered. }\end{array}$ & Uses RxNorm codes to identify medications \\
\hline MedicationOrder.reasonCodeableConcept & Reason for writing prescription & $\begin{array}{l}\text { Can check for opioids used for chronic pain } \\
\text { (SNOMED-CT code) }\end{array}$ \\
\hline MedicationOrder.dosageInstruction.dose[x] & Gives dose and units (ie $5 \mathrm{ml}$ ) & Used for MME calculator \\
\hline MedicationOrder.dosageInstruction.timing & $\begin{array}{l}\text { Gives how many times daily } \\
\text { (timing can be coded by } \\
\text { http://hl7.org/fhir/timing- } \\
\text { abbreviation) }\end{array}$ & Used for MME calculator \\
\hline Resource Type: & \multicolumn{2}{|c|}{ Diagnostic Report } \\
\hline Definition: & \multicolumn{2}{|c|}{$\begin{array}{l}\text { Findings and interpretations of diagnostic tests performed on patients. The } \\
\text { report includes clinical context such as requesting and provider information }\end{array}$} \\
\hline Elements & Definition & Use in App \\
\hline DiagnosticReport.code & $\begin{array}{l}\text { Codes that describe Diagnostic } \\
\text { reports }\end{array}$ & $\begin{array}{l}\text { Urinary Drug Screen (using LOINC code) } \\
\text { ordered }\end{array}$ \\
\hline DiagnosticReport.result & References Observation & \\
\hline Resource Type: & \multicolumn{2}{|r|}{ Observation } \\
\hline Definition: & \multicolumn{2}{|c|}{$\begin{array}{l}\text { Simple name/value pair assertions for laboratory data and other results such as vital } \\
\text { signs, imaging results, and social history. }\end{array}$} \\
\hline Elements & Definition & Use in App \\
\hline Observation. Patient & $\begin{array}{l}\text { A link to a resource representing } \\
\text { the patient to whose lab values } \\
\text { they are. }\end{array}$ & \\
\hline Observation. Code & $\begin{array}{l}\text { Codes identifying names of } \\
\text { simple observations; Use } \\
\text { LOINC or SNOMED codes }\end{array}$ & Use LOINC codes for UDS results \\
\hline
\end{tabular}




\begin{tabular}{|c|l|l|}
\hline Observation. Date & $\begin{array}{l}\text { Date range into which the } \\
\text { observation falls }\end{array}$ & $\begin{array}{l}\text { Recent Urinary Drug Screen or screen from } \\
\text { history }\end{array}$ \\
\hline Observation. Encounter & $\begin{array}{l}\text { Identifies the medication being } \\
\text { administered. }\end{array}$ & Uses RxNorm codes to identify medications \\
\hline Observation. Value[x] & $\begin{array}{l}\text { Information determined as a } \\
\text { result of making the } \\
\text { observation .value Quantity } \\
\text { gives a value; .Code able } \\
\text { Concept gives a code; .value } \\
\text { String gives a string output like } \\
\text { "Positive" or "Negative" }\end{array}$ & $\begin{array}{l}\text { Can check for Positive and negative results from } \\
\text { UDS }\end{array}$ \\
\hline
\end{tabular}




\subsection{SMART on FHIR}

SMART on FHIR is an open access standard based technology platform that creates apps that can run across different healthcare systems using the FHIR standards. With this interoperable system, patients, clinicians and healthcare providers can easily and efficiently access a library of apps to improve care based on personalized and precision medicine standards [8].

Unlike other CDS systems, SMART on FHIR focuses on creating an open and interoperable based technology platform, using FHIR standards, that allows vendor independent third-party apps to run securely using EHR data [8]. A publicly accessible app gallery links to dozens of clinical applications built on this platform that have been providing care at healthcare institutions such as Boston Children's Hospital and Duke Medicine. SMART on FHIR platform has even been expanded to include genomic data standards to unify how genomic variant data are accessed from multiple sequencing systems $[8,13]$.

SMART on FHIR has multiple components which include adaption of FHIR standards, authorization and authentication. Authorization is done using OAuth2, a Web standard for authorization whose key function is to enable an end user to approve a SMART app to access an EHR. Authentication is accomplished through OpenID Connect, a Web standard for authentication. It defines an OAuth2-based protocol allowing end users to sign into apps using external identity providers. The SMART on FHIR system, a health IT system that has implemented all of these components, can then run against a SMART on FHIR app. SMART on FHIR profiles require data to be coded using Meaningful Use terminologies and express constraints such as terminology restrictions, element cardinality restrictions, data type choice restrictions, and hierarchical structuring of resources [8]. Source code and examples of implementation are publicly available [3]. In addition, sandboxes, secure virtual testing environments that mimic a live EHR, are available through outlets such as Cerner's open developer $[7,16]$.

\subsection{Ontologies and Terminologies}

FHIR is designed to work with over 40 standardized terminologies and ontologies widely used in the biomedical informatics community [17]. See table 2. This project uses SNOMED-CT, RxNorm and LOINC. SNOMED CT is a standardized vocabulary for clinical terms used by physicians and other health care providers for the electronic exchange of medically-relevant health information [18]. In addition to providing thesaurus-capability for interlinking other coding systems and terminologies, SNOMED-CT provides a taxonomy, which is encoded in an ontological format. Elkin et. al [19] used the Mayo Clinic Vocabulary Server (MCVS) to successfully map free text clinical concepts to SNOMED -CT codes with a positive predictive value of 99.8 percent. RxNorm provides a standardized coding system for drugs, linking RxNorm identifiers to multiple other drug vocabularies [20]. RxNorm also provides some semantic structure by separating drug formulations from ingredients and separating brand names from clinical names, and so on. However, there is substantial overlap amongst codes, with no taxonomic way of grouping together the functional characteristics of drug families, such as the benzodiazepines. LOINC is a common reference terminology for clinical and laboratory measurements, assays, patient information, and so on [21]. However, unlike RxNorm, LOINC does not have any taxonomic structure encoded in way that allows machine reasoning as is possible with an ontological implementation. Neither terminology 
links codes via formalized relations that support moving between levels of granularity in any systematic way.

This is problematic for our project due to the large of number of potential codes we need to search on discover the prescription of opioids. Over 13,000 codes for various forms of opioids alone, not including benzodiazepines, and related drugs like muscle relaxants. There exists no subset of codes that would map to the entire value set constructed of drugs with the general opioid characteristics for potential of abuse. Enumerating all the potential codes is possible, even could be done automatically with the right scripting tools. However, it is not an extensible or easily updated methodology. If this application were to be effectively deployed in a clinical environment, a much more robust way of coding the resource requests would be required. At a minimum, the high-level logic of the decision tree would need to decouple form the particular value sets the enumerate all the possible drug and procedure codes

Table 2: List of standard terminologies available in FIHR

\begin{tabular}{|l|l|}
\hline Name of Standard & Name of Standard \\
\hline SNOMED CT & IETF language \\
\hline RxNorm & $\begin{array}{l}\text { Mime Types Multipurpose Internet Mail } \\
\text { Extensions }\end{array}$ \\
\hline LOINC & $\begin{array}{l}\text { Medical Device Codes defined in ISO } \\
11073-10101\end{array}$ \\
\hline UCUM: UnitsOfMeasure & DICOM Code Definitions \\
\hline NCI Metathesaurus & Health Canada Drug Identification Number \\
\hline AMA CPT codes & NUCC Provider Taxonomy \\
\hline NDF-RT National Drug File - Reference & $\begin{array}{l}\text { HGNC: Human Gene Nomenclature } \\
\text { Committee }\end{array}$ \\
\hline Terminology & ENSEMBL reference sequence identifiers \\
\hline NDique Ingredient Identifier (UNII) & $\begin{array}{l}\text { REFSEQ: National Center for Biotechnology } \\
\text { Information (NCBI) }\end{array}$ \\
\hline CVX (Vaccine Administered) & ClinVar \\
\hline ISO 2 letter Country Codes & Sequence Ontology \\
\hline NUBC code system for Patient Discharge & HGVS: Human Genome Variation Society \\
\hline Status & $\begin{array}{l}\text { DBSNP: Single Nucleotide Polymorphism } \\
\text { database }\end{array}$ \\
\hline RadLex & $\begin{array}{l}\text { COSMIC: Catalogue Of Somatic Mutations } \\
\text { In Cancer }\end{array}$ \\
\hline ICD-9 \& ICD-10 &
\end{tabular}




\begin{tabular}{|c|c|}
\hline $\begin{array}{l}\text { ICPC International Classification of Primary } \\
\text { Care }\end{array}$ & LRG: Locus Reference Genomic Sequences \\
\hline $\begin{array}{l}\text { ICF Classification of Functioning, Disability } \\
\text { and Health }\end{array}$ & $\begin{array}{l}\text { OMIM: Online Mendelian Inheritance in } \\
\text { Man }\end{array}$ \\
\hline Version 2 tables & PubMed \\
\hline A HL7 v3 code system & $\begin{array}{l}\text { PHARMGKB: Pharmacogenomics } \\
\text { Knowledge Base }\end{array}$ \\
\hline $\begin{array}{l}\text { Anatomical Therapeutic Chemical } \\
\text { Classification System }\end{array}$ & ClinicalTrials.gov \\
\hline $\begin{array}{l}\text { Anatomical Therapeutic Chemical } \\
\text { Classification System }\end{array}$ & European Bioinformatics Institute \\
\hline
\end{tabular}

FHIR allows for the use of Concept Maps that allow for the linking of one or more codes (taken here to be concepts) to one or more codes in another system. The mappings use equivalence properties, similar to the kinds of synonymy relations found in thesauri, such as narrower, wider, specializes. In addition to mapping between concepts (codes), FIHR allows for the mapping between one or more concepts and a value set. This would allow for the trigger for query on Medication Order to use a generic set of codes to map to a much broader set of enumerated RxNorm codes for all relevant opioids. However, it still doesn't alleviate the problems surrounding the maintenance and generation of that list as the guidelines or coding systems change.

\subsection{Proposed Development}

A hybrid HTML5 app is currently being built using the SMART JavaScript client library, an open library designed to assist with calling the FHIR API and handling the SMART on FHIR authorization and authentication workflow [4]. A HTML5 app was chosen for cross-platform use and to facilitate the app within the EHR environment by running it through a browser widget. Native iOS and Android applications may be developed in the future.

In order to access the FHIR resources in the EHR through the EHR's web services, the app will use the following process. When a clinician wants to use OPIacutepain to assess the risk of prescribing, the EHR redirects to the SMART launch URI, implemented in the file launch.html, then redirects to the FHIR authorization server, and then after a successful authentication, redirects to the file index.html. The FHIR authorization server can then be accessed using the fhir-client.js file and calling FHIR.oauth2.authorize in the launch.html file with the client id. The authorization code is then exchanged for an access token to the authorization server using the FHIR client. When Index.html is invoked, the SMART application will have the ability to request FHIR resources from the EHR for the patient to run the SMART application.

The FHIR JavaScript client also facilitates calling and searching code able concepts through the function by Codes to find our medication list through coded terms. Finally, the visual form of the application can be called from draw Visualization, a built in function that uses the identification 
placeholders defined in index.html. This file contains the format for the output of the app. The displayed output for the clinician within the app will contain the MME/day risk calculator when it can be calculated based on listed medications, whether or not a previous UDS was ordered and the result, a table of medications for benzodiazepines and opioid medications, and patient identification details, including gender, date of birth, and condition. Finally, the appropriate patient specific recommendation based on the guidelines presented by the CDC and dependent on the outcomes from the medication lists, urine drug testing results, and MME/day calculator will be displayed for the clinician within the app.

Usability testing will be done following the creation of the app to ensure that it adds to the workflow process of the clinician and is designed efficiently and productively for clinicians who frequently prescribe opioid medications.

\section{Discussion and Conclusion}

Prescription opioid pain medications are a big contributor to the opioid epidemic. Attempts have been made in the past to create CDSS tools within the EHR to aid clinicians in prescribing and managing patients with chronic pain. The effectiveness of CDSS depend on how well they are integrated and how easy it is to access them.

The SMART on FHIR platform addresses both ease of access while maintaining safety and security of sensitive patient information through an authorization process. It also gives feedback based on personalized patient information. This type of third-party CDSS may be able to improve quality of care through precision medicine. In addition, there is no user training required as the app is able to collect the back ground information automatically and provide it on demand through a secure authentication process. The FHIR interoperability standards makes this usable by all EHRs that are compatible with HL7 FHIR standards.

\section{Limitations}

This OPIpain SMART on FHIR app is an open source interoperability standard based third party app that has not been tested and is a work in progress. The template that it is built on is new and has not received much feedback on its usability and effectivity. The outcome measure of these apps would take longer. Taking this background into consideration the following aspects would have to be taken into account in further developing and implementing this app.

1. The accuracy of the EHR-specific logic to transform the specific data structures to corresponding FHIR resources and with SMART specific profiles so that it is able to pull up the proper information for this on demand app.

2. The SMART on FHIR apps that have been used so far have not been able to validate the data that they have been pulling through the app. It is a work in progress and can only be evaluated on implementation.

3. Semantic parsing of terminologies through FHIR would be based on the concept mapping which relies on how good the terminologies and codes are. 
The interventions for regulating and monitoring prescription pain medications and optimizing pain management cannot be delayed till there are better methods of concept mapping. Hence it would only be as good as the current mapping standards.

\section{References}

1. Rudd RA. 2016. Increases in drug and opioid-involved overdose deaths - united states, 20102015. MMWR Morbidity and Mortality Weekly Report. 65. 2017 Feb;20(2S):S3-S92. PubMed PMID: 28226332.

2. Dowell D, Haegerich TM, Chou R. 2016. CDC guideline for prescribing opioids for chronic pain-united states, 2016. JAMA. 315(15), 1624-45. PubMed https://doi.org/10.1001/jama.2016.1464

3. Mandel JC, Kreda DA, Mandl KD, Kohane IS, Ramoni RB. 2016. SMART on FHIR: A standards-based, interoperable apps platform for electronic health records. J Am Med Inform Assoc. 23(5), 899-908. Epub Feb 2016. doi:https://doi.org/10.1093/jamia/ocv189. PubMed

4. Manchikanti L,et. al. 2017 Responsible,Safe, and Effective Prescription of Opioids for Chronic Non-Cancer Pain: American Society of Interventional Pain Physicians (ASIPP) Guidelines. Pain Physician.

5. Federal Health IT website: CDSS guidelines: https://www.healthit.gov/sites/default/files/clinical-decision-support-0913.pdf

6. New York Consolidated Laws, Public Health Law - PBH § 3331. Scheduled substances administering and dispensing by practitioners - http://codes.findlaw.com/ny/public-healthlaw/pbh-sect-3331.html

7. Lesselroth BJ, Yang J, McConnachie J, et al; Addressing the sociotechnical drivers of quality improvement: a case study of post-operative DVT prophylaxis computerised decision support

8. Haffey F, Brady RR, Maxwell S. 2013. A comparison of the reliability of smartphone apps for opioid conversion. Drug Saf. 36(2), 111-17. PubMed https://doi.org/10.1007/s40264$\underline{013-0015-0}$

9. Guideline Resources. CDC Opioid Guideline Mobile App; https://www.cdc.gov/drugoverdose/prescribing/app.html

10. $\mathrm{pH}$ - Medical Opioid Converter by Philip Eagan; https://itunes.apple.com/us/app/phmedical-opioid-converter/id1082147868?mt=8 
11. Safe Opioids app aims to prescribe narcotics more appropriately with patients; by Douglas Maurer, DO/MPH/FAAFP | February 1, 2016

https://www.imedicalapps.com/2016/02/safe-opioids-prescribing-narcotics- patients/

12. OpioidCalcNYC App.

https://play.google.com/store/apps/details?id=gov.nyc.health.MME\&hl=en

13. Wilsey BL, Fishman SM, Casamalhuapa C, Gupta A. 2009. Documenting and improving opioid treatment: the Prescription Opioid Documentation and Surveillance (PODS) System. Pain Med. 10(5), 866-77. Epub Jul 2009. doi:https://doi.org/10.1111/j.1526$\underline{\text { 4637.2009.00652.x. }}$ PubMed

14. Using FHIR to develop a healthcare mobile application. Wireless Mobile Communication and Healthcare (Mobihealth), 2014 EAI 4th International Conference on; 2014: IEEE.

15. FHIR. 2017. HL7.org: https://www.hl7.org/fhir/overview.html.

16. Millenium. 2017. CernerCode: Cerner code: http://fhir.cerner.com/millennium/dstu2/

17. Alterovitz G, Warner J, Zhang P, Chen Y, Ullman-Cullere M, et al. 2015. Smart on fhir genomics: Facilitating standardized clinico-genomic apps. J Am Med Inform Assoc. •••, ocv045. PubMed https://doi.org/10.1093/jamia/ocv045

18. SNOMED IHTSDO. (2017) https://www.nlm.nih.gov/healthit/snomedct/index.html

19. Elkin PL, Brown SH, Husser CS, Bauer BA, Wahner-Roedler D, et al. 2006. Evaluation of the Content Coverage of SNOMED CT: Ability of SNOMED Clinical Terms to Represent Clinical Problem Lists. Mayo Clin Proc. 81(6), 741-48. PubMed https://doi.org/10.4065/81.6.741

20. RxNorm NLM. UMLS (2017) https://www.nlm.nih.gov/research/umls/rxnorm/

21. 1998. Huff SM etal. (1998) Development of the Logical Observation Identifier Names and Codes (LOINC) vocabulary. J Am Med Inform Assoc. 5(3), 276-92. PubMed https://doi.org/10.1136/jamia.1998.0050276

22. Manchikanti L,et. al. 2017 Responsible,Safe, and Effective Prescription of Opioids for Chronic Non-Cancer Pain: American Society of Interventional Pain Physicians (ASIPP) Guidelines. Pain Physician.

\section{Acknowledgements/Grants}

This work has been supported in part by an NIH NCATS Clinical and Translational Science Award (UL1TR001412-01) and an NIH T32grant (T32 GM099607) from the Department of Anesthesiology. 


\section{Appendix 1: OPIacutepain: flow diagram}
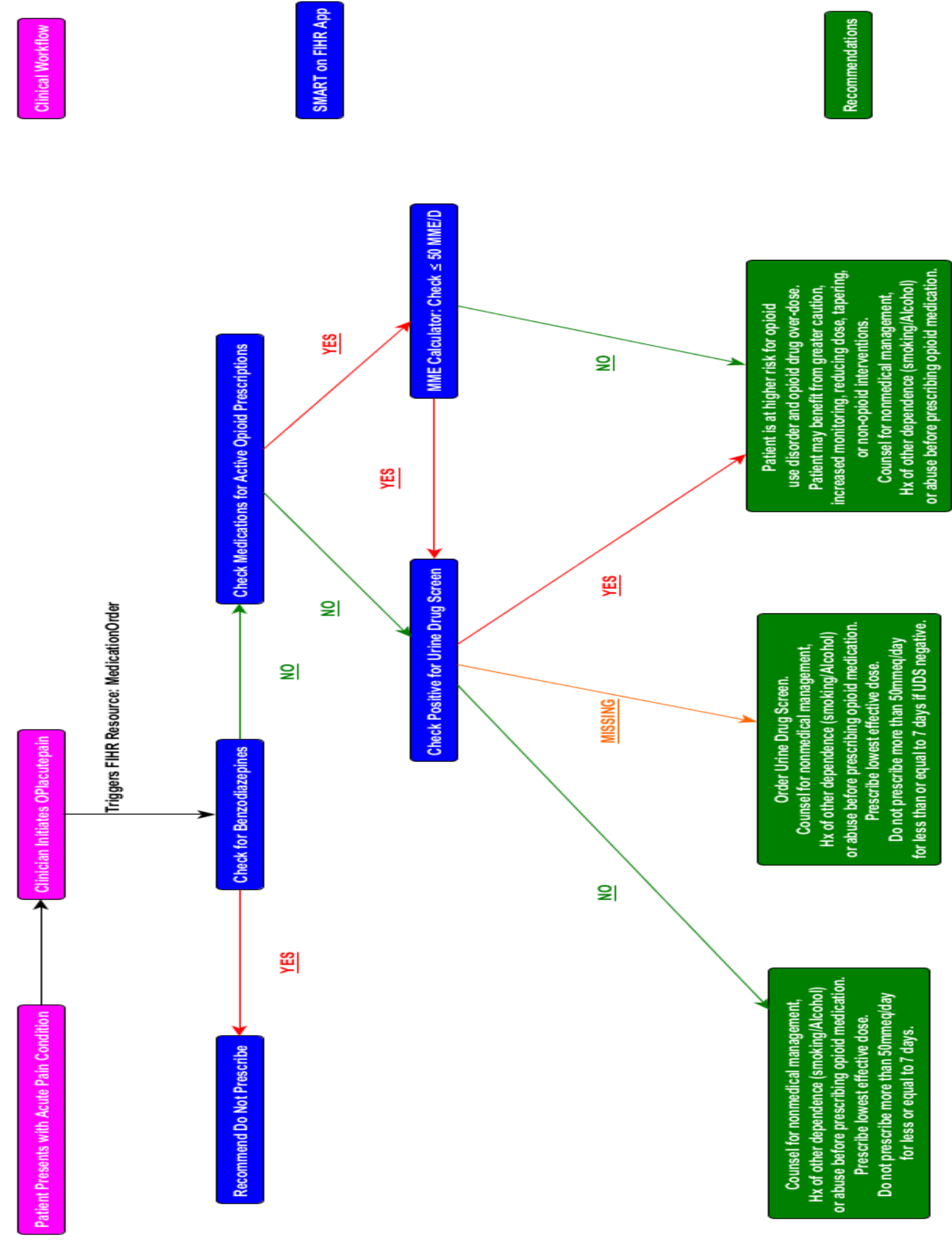

Online Journal of Public Health Informatics * ISSN 1947-2579 * http://ojphi.org * 9(2):e193, 2017 